\title{
Störungen der Bluthirnschranke bei gedeckten stumpfen Schädel-Hirntraumen
}

\author{
Von J. Gerlach und H. Becker \\ Aus der Chirurgischen Universitätsklinik Würzburg, Direktor: Professor Dr. W achs muth, \\ und dem \\ Max-Planck-Institut für Hirnforschung Gießen, Direktor: Professor Dr. S p a t z. \\ (Z. Naturforschg. 8 b, 578-581 [1953]; eingegangen am 13. Juli 1953)
}

Herrn Professor Dr. Hallervorden zum 70. Geburtstag

\begin{abstract}
An 19 Katzen wurde experimentell das Verhalten der Bluthirnschranke nach gedeckter stumpfer Hirnverletzung untersucht. Die Schrankenprüfung erfolgte mit dem Farbstoff Astraviolett FF nach dem Verfahren von B e cke r und Q u a d b e ck, die Traumatisierung wurde in Anlehnung an das Vorgehen von Denny-Brown vorgenommen. Schon bei geringfügigen Schädelhirntraumen stellte sich eine erhebliche Störung der Bluthirnschranke heraus, deren Grad von der Stärke des Traumas und der Zeit zwischen Trauma und Schrankenprüfung abhängt. Die Schrankenstörung betraf nicht nur das Gehirn, sondern in vielen Fällen auch das Rückenmark. Sie war am meisten ausgeprägt in den Grenzzonen der Versorgungsgebiete der Hirngefäße. Als Ursache der Schrankenstörung werden Gefäßregulationsstörungen mit Hypoxydose und Gewebs-Säuerung angenommen. Auf die klinische Bedeutung der Befunde wird hingewiesen.
\end{abstract}

$\mathrm{D}$ ie Pathophysiologie der gedeckten traumatischen Hirnschädigung ist erst in den letzten Jahrzehnten genauer studiert worden. Auf diesem Gebiet ist ebenso wie auf dem der Anatomie und Klinik ein umfangreiches Schrifttum entstanden, auf das wir hier nicht näher eingehen wollen.

In der vorliegenden Mitteilung beschäftigen wir uns mit einem einzelnen, theoretisch und praktisch bedeutungsvollen pathophysiologischen Problem des gedeckten Hirntraumas, nämlich mit dem der posttraumatischen Störungen der Bluthirnschranke. Die Untersuchung dieser Frage lag um so näher, als das Verhalten der Blutliquorschranke beim Hirntrauma bereits mehrfach geprüft worden ist, wobei eine vermehrte Durchlässigkeit dieser Barriere festgestellt wurde $^{1}$. Bislang fehlte es jedoch an einer für die Bearbeitung unserer Fragestellung geeigneten Methode. B e c k e r und Q u a d b e c k ${ }^{2}$ haben nun kürzlich ein Verfahren zur Prüfung der Bluthirnschranke entwickelt, mit dem im Tierexperiment diese Schwierigkeit überwunden wird. Für die Begründung der Methode sei auf die Arbeit der genannten Verfasser, für die allgemeinen Grundlagen im Falle der Hirn-

1 M. S. Z e t t e r h o lm, Acta psychol. neurol., Suppl. XLV [1947]; D. E n g e l, Acta psychiatr. 53, 231 [1948]; D. Z a nello, Riform. med. 1635 [1938], cit. Z. ges. Chirurgie 92, 664 [1939]; S. Caccuri, Policlinico. sez. med. 32, 309 [1925], cit. n. Z. ges. Chirurgie 33, 199 [1926]. volumenvermehrung auf unsere vorangegangene Mitteilung ${ }^{4}$ verwiesen. In der letztgenannten Arbeit konnten wir die schon lange gehegte Vermutung bestätigen, daß Hirnvolumenvermehrungen ganz allgemein mit Permeabilitätsstörungen verbunden sind. Die spezielle Aufgabe der vorliegenden Arbeit war, das längst bekannte Auftreten der Hirnvolumenvermehrung im Sinne posttraumatischen Hirnödems auf seine Beziehungen zu Störungen der Schrankenfunktion hin zu untersuchen.

\section{Material und Methodik}

Die Versuche wurden an 19 Katzen ausgeführt. Die Traumatisierung wurde in Anlehnung an das Vorgehen von Denny-Brown ${ }^{3}$ mittels eines Pendels von $195 \mathrm{~cm}$ Länge vorgenommen, das mit einem Gewicht von $2,5 \mathrm{~kg}$ belastet war; vereinzelt wurde der elastisch fixierte Kopf durch Hammerschläge traumatisiert. Zum Teil verzichteten wir auf eine medikamentöse Vorbereitung, z. Tl. erhielten die Tiere zur Ruhigstellung vor dem Trauma 10 E Curarin/kg; um die Wirkung des Traumas klinisch bestimmen zu können, mußten wir von einer Narkose absehen. Die Fallhöhe des Pendels wechselte zwischen 20 und $110 \mathrm{~cm}$. Die damit erzielten Schädel-Hirntraumen waren überwiegend leichter bis gelegentlich mittelschwerer Natur, erfüllten jedoch die Bedingungen nicht, die Denny-Brown an eine experimentelle Commotio

2 H. B e cker u. G. Qu a d b e ck, Z. Naturforsch. 7 b, 493 [1952].

3 Denny-Brown, Physiol. Rev. 125, Nr. 2 [1945]. 
stellt; so haben wir in keinem Fall einen Verlust des Hornhautreflexes gesehen. Die Methodik der Schrankenprüfung ist in der erwähnten Arbeit von B e c ker und $\mathrm{G}$ e r l a c h ${ }^{4}$ genau beschrieben; sie kam in gleicher Weise zur Anwendung.

Astraviolett $\mathrm{FF}^{*}$ ist ein basischer Farbstoff, der die intakte Bluthirnschranke nicht zu durchdringen vermag, bei nur geringfügigen Störungen des $p_{\mathrm{H}^{-}}$Gleichgewichtes zwischen Blut und Hirngewebe jedoch durch die Endothelmembran hindurchtritt und zu einer Rot-Violett-Färbung des Gehirns führt. Die Injektion erfolgt, in willkürlich gewählten Zeitabständen nach dem experimentellen Eingriff, intracardial; sofort anschließend wird der noch in den Gefäßen befindliche Farbstoff auf dem gleichen Wege mit Formalin ausgespült.

\section{Ergebnis se}

Wir gruppieren unsere Befunde nach der Schwere des Traumas und nach der Überlebenszeit der Tiere. Das Trauma war in keinem Falle die Todesursache. Die Úberlebenszeit wurde jedesmal willkürlich festgesetzt. Klinisch zeigten alle Tiere keine Auffälligkeiten.

\section{Gruppe. Geringfügige Traumen}

1. Fallhöhe des Pendels $25 \mathrm{~cm}$, Überlebenszeit 22 Min. Es findet sich ein cerebellarer Druckkonus als Zeichen des erhöhten Hirndrucks, das ganze Gehirn weist einen schwach rötlichen Farbstich auf, vor allem, zugleich mit Petechien und Farbspritzern, in den Grenzgebieten der Versorgungszonen der Hirngefäße (Grenzzonen); s. Abb. 1a ${ }^{* *}$. In die Rotfärbung sind weiße Flecken eingestreut. Das Rückenmark zeigt eine bis zum caudalen Ende spurenweise (?) Anfärbung. Auch auf den Schnitten durch das Gehirn treten die Grenzzonen deutlicher gefärbt hervor. Während das Hemisphärenmark und der Thalamus farbfrei sind, finden wir den Kleinhirnwurm und den Konus fleckig betont.

2. Fallhöhe des Pendels $25 \mathrm{~cm}$, Überlebenszeit $3 \mathrm{Stdn}$. 30 Min., 2 Pendelschläge.

Auch dieses Gehirn läßt einen deutlichen Druckkonus erkennen. Die Gehirnoberfläche ist violettstichig gefärbt, mit eingesprengten weißen Flecken, vor allem auf dem Kleinhirn. Die Grenzzonen sind sehr stark herausgehoben. Das Rückenmark ist ebenfalls bis zum Caudalende entsprechend gefärbt. Auf den Schnitten treten die Grenzzonen hervor, der Thalamus ist stark gefärbt, auch das Mark zeigt einige Farbspritzer. Wiederum ist der Kleinhirnwurm lebhaft rot gefleckt.

3. Fallhöhe $25 \mathrm{~cm}$, Überlebenszeit 7 Stdn.; Gehirn und Rückenmark ungefärbt.

4. Fallhöhe $25 \mathrm{~cm}$, Überlebenszeit 21 Stdn.; keine Färbung von Hirn und Rückenmark.

* Neuerdings benutzen wir an Stelle des nicht mehr lieferbaren Astraviolett FF den auch jetzt noch erhältlichen nahe verwandten Farbstoff Astraviolett FD, der an der Schranke die gleichen Eigenschaften zeigt.
II. Gruppe. Stärkere Traumen a

5. Zwei Schläge, Fallhöhe 50 bzw. $80 \mathrm{~cm}$; Überlebenszeit 20 Minuten.

Das Gehirn zeigt keinen Konus und keine Volumenvermehrung. Die Oberfläche ist blaßviolett mit geringer Betonung der Grenzzonen; dort kleine petechiale Blutungen. Das Rückenmark ist nur fraglich gefärbt. Auf den Schnitten heben sich lediglich der Gyrus lateralis und die Kniehöcker durch stärkere Färbung ab.

6. Fallhöhe $50 \mathrm{~cm}$, Überlebenszeit 25 Minuten.

An diesem Gehirn findet sich ein starker Druckkonus. Die Hirnoberfläche weist allgemein einen sehr geringen Farbstich auf, aber sehr starke Flecken in den Grenzzonen. Das Kleinhirn ist nur angedeutet fleckig (Abb. 1d). Das Rückenmark ist bis nach caudal geringfügig angefärbt. Auf den Schnitten sieht man leichte Diffusfärbung. Die Grenzzonen sind deutlich fleckig rot, der Hirnstamm weniger gefleckt, das Mark farblos.

\section{Gruppe. Stärkere Traumen b}

7. Fallhöhe $110 \mathrm{~cm}$, Überlebenszeit 15 Min., das Pendel traf ungenau.

Das Gehirn ist am schwächsten von allen Traumahirnen gefärbt. Die Grenzzonen sind durch diffuse Färbung und Farbflecken deutlich herausgehoben (Abb. 1 b). Das Rükkenmark ist ungefärbt. Die Schnitte zeigen schwache Diffusfärbung mit Betonung des Gyrus lateralis, der Schläfengegend, der Vierhügel, des Wurmes und der Kleinhirntonsillen.

8. Fallhöhe $110 \mathrm{~cm}$, Überlebenszeit 15 Minuten.

Das Gehirn ist im ganzen stärker gefärbt als das vorige, jedoch sind die Grenzzonen weniger betont, die Farbflecken kleiner. Das Rückenmark findet sich bis nach lumbal schwach durchgefärbt. Auf den Schnitten erkennt man schwache diffuse Färbung ohne Prädilektion. Das Rautenhirn zeigt den gleichen Farbton wie das Großhirn.

9. Fallhöhe $110 \mathrm{~cm}$, Überlebenszeit 20 Minuten.

Das Gehirn ist bis zum Halsmark einschließlich gleichmäßig violett gefärbt. Weiter caudal ist das Rückenmark wegen Aortenligatur bei der Spülung nicht mehr zu beurteilen. Auf den Schnitten sind alle Grisea gleichmäßig gefärbt, nur das Geniculatum laterale ist herausgehoben.

10. Fallhöhe $110 \mathrm{~cm}$, Überlebenszeit 20 Minuten.

Bei dieser Katze ist das Gehirn „marmoriert“ und bietet neben ganz dunkelroten Stellen fast ungefärbte Bezirke (Abb. 1 c), das Rückenmark ist bis zum caudalen Ende intensiv durchgefärbt. Die Hirnschnitte ergeben ein sehr buntes Bild. Die Gyri laterales und suprasylvii sind stark gefärbt, während sich seitlich ein weitgehend ungefärbtes Gebiet anschließt. Auf den Schnitten sieht man die Stammganglien fleckig, den Thalamus dunkel, den Hypothalamus blaß, die Vierhügel gefärbt, den Nucleus ruber und die Substantia nigra blaß, den Kleinhirnwurm stark, die Kleinhirnhemisphäre weniger gefärbt, das Rautenhirn blaß (Abb. 2).

4 H. B e c ker u. J. G e r l a c h, Z. ges. exp. Med., im Druck.

** Abb. $1 \mathrm{a}-\mathrm{d}$ u. 2, s. Tafel S. 580 a. 
11. Fallhöhe $110 \mathrm{~cm}$, Überlebenszeit 1 Stde. 4 Min.

Es findet sich ein starker Druckkonus und eine nur schwach violette Färbung der Hirnoberfläche ohne Farbflecken. Das Rückenmark ist bis caudal schwach angefärbt. Die Schnitte zeigen gleichmäßig geringe Färbung.

12. Fallhöhe $110 \mathrm{~cm}$, Überlebenszeit 2 Stdn. 15 Min. Der Schlag traf nicht ganz genau.

Ein Druckkonus fehlt. Die Hirnoberfläche ist violettstichig mit mäßigen Farbflecken in den Grenzzonen und im Kleinhirn. Das Rückenmark ist durchgefärbt. Auf den Schnitten findet sich geringe Diffusfärbung, Thalamus und Kleinhirn sind etwas fleckig.

13. Fallhöhe $110 \mathrm{~cm}$, Überlebenszeit 5 Stunden.

Das Gehirn zeigt einen starken Druckkonus. Sein Farbstich ist sehr gering und nur im Vergleich zu ungefärbten Gehirnen sichtbar. Das Rückenmark ist nicht sicher gefärbt. Auf den Schnitten finden sich Farbspuren in den Grisea, vor allem im Thalamus und im Kleinhirn.

14. Fallhöhe $110 \mathrm{~cm}$, Überlebenszeit 13 Stunden.

Ein Druckkonus ist angedeutet. Die Hirnoberfläche zeigt einen geringen Violettstich sowie wenige Farbflecken in den Grenzzonen. Das Rückenmark ist nicht sicher gefärbt. Auf den Schnitten sieht man schwache diffuse Färbung mit Flecken im Gyrus lateralis und teilweise in der ersten Bogenwindung.

15. Fallhöhe $110 \mathrm{~cm}$, Überlebenszeit 23 Stunden. Zwei Schläge. Klinisch Mydriasis, verbunden mit vorübergehender Schwäche des rechten Hinterbeines.

Das Gehirn ist deutlich violettstichig ohne bevorzugte Regionen.

16. Fallhöhe $110 \mathrm{~cm}$, Überlebenszeit 48 Stunden.

Ein Druckkonus ist hier deutlich. Die Hirnoberfläche weist eine gleichmäßige mittelviolette Färbung auf. Das Rückenmark ist bis zum Konus gefärbt. Die Schnitte zeigen eine deutliche diffuse Färbung aller Grisea.

17. Fallhöhe $110 \mathrm{~cm}$, Überlebenszeit 72 Stunden.

Das Gehirn zeigt einen deutlichen Druckkonus und einen nur sehr geringen Farbstich. Einige nur blaßviolette Flecken befinden sich an den Grenzzonen und am Kleinhirn. Das Rückenmark ist bis caudal gefärbt. Auf den Schnitten findet sich eine diffuse schwache Färbung der Grisea, wobei Caudatum und Thalamus mehr betont sind als die Rinde.

\section{Gruppe. Starke Traumatisierung des Gehirns}

18. Drei Hammerschläge auf das linke Scheitelbein. Danach kommt es zu Mydriasis und Pupillenstarre, das Tier mauzt nach einigen Sekunden und verkriecht sich. Lähmungen bestehen nicht. Überlebenszeit 24 Stunden.

Man findet einen Schädelbruch links frontal am Hinterende der Stirnhöhle mit einer $3 \mathrm{~mm}$ langen, $2 \mathrm{~mm}$ tiefen Impression, ferner eine Blutung in die Stirnhöhle. Am Gehirn sind keine Kontusionsherde und keine Blutungen festzustellen. Die linke Hemisphäre ist sicher verbreitert, vor allem die erste Bogenwindung links occipital ist $1 \mathrm{~mm}$ breiter als rechts. Die Hirnkammern sind von links nach rechts über die Mittellinie verschoben. Die Groß- und Kleinhirnoberfläche ist gleichmäßig mittelviolett gefärbt, links etwas mehr als rechts, vor allem im Gyrus suprasylvius. Das Rückenmark läßt starke Anfärbung der grauen Substanz bis zum Sakralmark erkennen. Auf den Schnitten finden sich das Rindengrau, die Stammganglien, der Thalamus sowie das Mittel- und Nachhirngrau erheblich angefärbt.

19. Ein Schlag mit dem Bleihammer auf das linke Scheitelbein ohne Reaktion, nach 3 Min. wird ein stärkerer Schlag ausgeführt. Danach mauzt das Tier, zeigt Nasenbluten und verkriecht sich. Beide Pupillen sind sehr weit und reagieren sehr träge auf Licht. Am nächsten Tag ist die Katze klinisch unauffällig. Úberlebenszeit 23 Stunden.

Bei der Schrankenprüfung kommt es vorübergehend zu einer Störung der Formalinspülung, da die Nadelspitze im Pericard hängenbleibt. Das Gehirn ist gut gehärtet bis auf die Occipitalpole, an denen sich auch noch eine Spur Gefäßfüllung zeigt. Die linke Stirnhöhle enthält Blut. Das Gehirn weist überall an den Grenzzonen und an beiden Occipitalpolen eine dunkelviolette Marmorierung auf, die links fast bis zum Frontalpol reicht. Die Färbung ist links lebhafter als rechts. Das Kleinhirn ist schwach violettstichig, ein Druckkonus ist fraglich. Auf den Schnitten sieht man leichte Diffusfärbung des ganzen Gehirns, in den Grenzzonen Bevorzugung der Täler. Die Färbung der Occipitalpole ist wegen der Störung bei der Spülung nicht verwertbar.

Besprechung der Versuchsergebnisse

Schon bei ganz geringfügigen Hirntraumen, bei denen die Bedingungen der Commotio, wie sie Denny-Brown und Rus se ${ }^{5}$ formuliert haben, noch nicht erfüllt sind, beobachten wir eine erhebliche Störung der Blut-Hirnschranke. Die genannten Autoren verlangen von einer experimentellen Commotio, daß das Trauma zur Aufhebung des Hornhautreflexes führt. Bei den leichtesten Traumen unserer Versuchsreihe war die Schrankenstörung nur einige Stdn. lang nachweisbar und verschwand zwischen der vierten und der siebenten Stde. nach dem Trauma, bei schwereren Traumen trat sie nach 72 Stdn. noch deutlich hervor. Sie war stets über das ganze Gehirn ausgebreitet, bei stärkerem Trauma auf der Seite der Gewalteinwirkung ausgeprägter und erstreckte sich bei längerer Überlebenszeit bzw. stärkerer Traumatisierung auch auf das Rückenmark. Diese Abhängigkeit der Farbintensität von der Überlebensdauer und der Stärke des Traumas gilt übrigens ganz allgemein für das gesamte Zentralnervensystem. Zum Unterschied von der Permeabilitätsstörung, die wir bei Hirnvolumenvermehrung aus anderen Ursachen beobachten, ist nach Hirntraumen die Bevorzugung der Grenzzonen ein hervorstechen-

5 Denny-Brown u. Russel, Brain 64, 93 [1941]. 


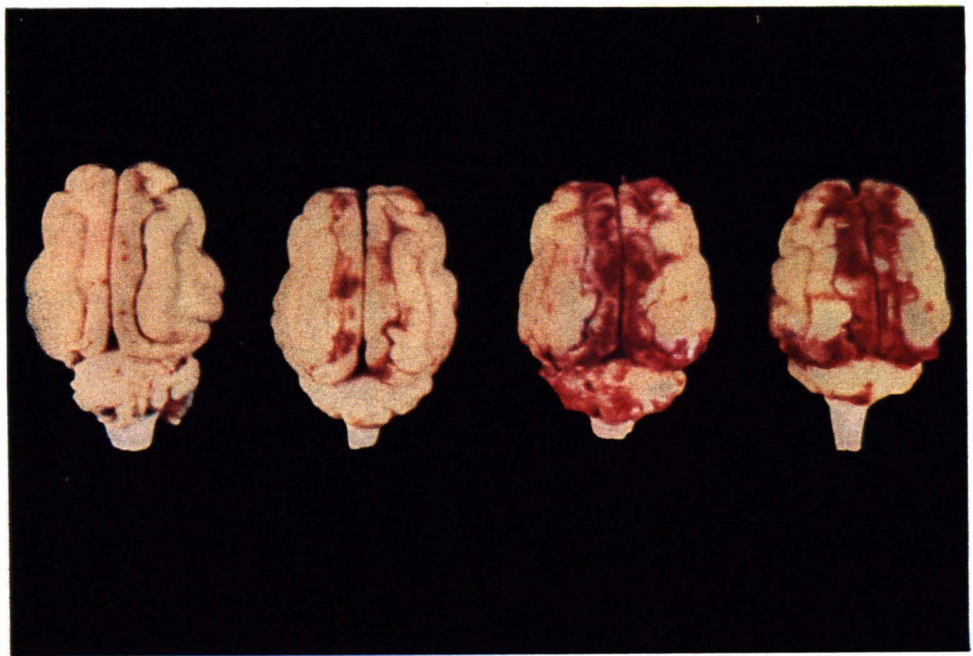

a

b

c

d

Abb. 1. Mit Astraviolett FF vitalgefärbte Katzengehirne nach verschieden starker Traumatisierung; Überlebenszeit z. Tl. unterschiedlich. a) Geringfügiges Trauma, Überlebenszeit 22 Min.; schwach rötliche Diffusfärbung, Petechien und Farbspritzer in den Grenzzonen. b) Stärkeres Trauma, Überlebenszeit 15 Min.; diffuse Rotfärbung fast nur in den Grenzzonen, dort auch stärkere Farbflecke. c) Stärkeres Trauma, 20 Min. Überlebenszeit. Stärkere rot-violette Färbung des ganzen Gehirns mit sehr starker Betonung der Grenzzonen. d) Schwächeres Trauma, Überlebenszeit $2.5 \mathrm{Min}$; ; ungefähr der gleiche Färbeeffekt wie bei c).

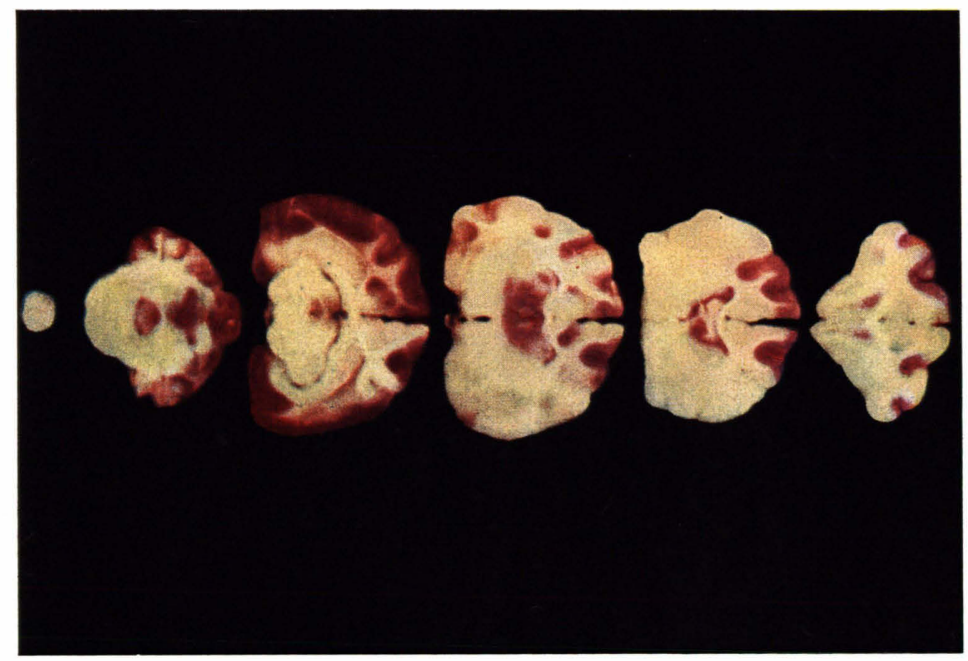

Abb. 2. Frontalschnitte durch das Gehirn von Abb. 1c; s. Text. 
O.Kratky, A.Sekora und E.Treiber, Neue Äquator-Kleinwinkelinterferenz an Polyvinylchlorid und Polyacrylnitril (S.615).

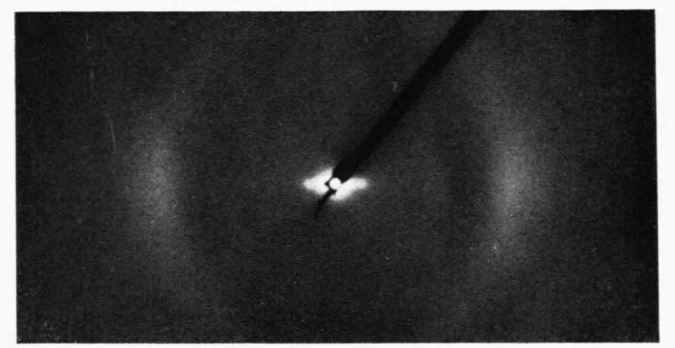

Abb. 1.

J.Jensen und E. Thofern, Chlorhämin (Ferriporphyrinchlorid) als Bakterienwuchsstoff (S. 599).

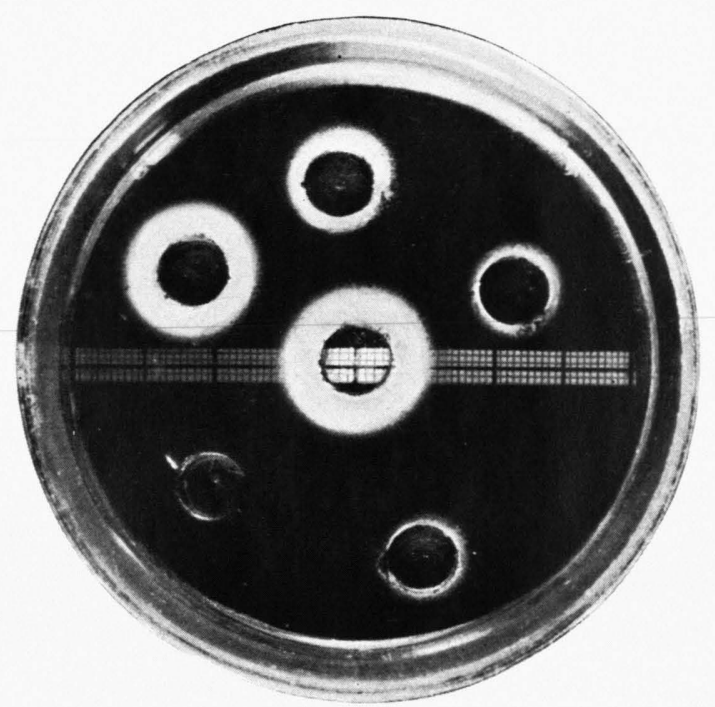

Abb. 1. Gleichmäßig mit Var. beimpfte Agaroberfäche nach 18-stdg. Bebrütung. Um die Stanzlöcher, die fallende Häminkonzentrationen enthalten (von $1,0 \times 10^{-4}$ in der Mitte über $5 \times 10^{-5} ; 8,5 \times 10^{-6} ; 6,25 \times 10^{-6}$ bis $3,1 \times^{-6} \mathrm{im}$ Uhrzeigersinn), sind die Wachstumszonen deutlich sichtbar. Das Loch ohne Zone enthält nur Phosphatpuffer. Die Dichte der Wachstumszonen und das kaum sichtbare Wachstum auf der übrigen Platte wird durch den Streifen Millimeterpapier, der unter der Petrischale liegt, veranschaulicht. 
des Merkmal. Zur Deutung dieser Erscheinung müssen u. E. die beim Trauma bekannten Gefäßregulationsstörungen herangezogen werden, die in den Grenzgebieten der arteriellen Versorgungsgebiete infolge der hier besonders großen vasomotorischen Labilität ausgeprägter sind als anderswo ${ }^{6}$. Da wir bei der histologischen Untersuchung die Gefäße in den Grenzzonen meist erheblich enger fanden als am übrigen Gehirn, ist es einerseits möglich, daß die Farbe bei der Durchspülung an diesen Stellen intravasal länger liegen bleibt und daß die beobachteten Farbflecken durch die postmortal einsetzende Durchlässigkeit der Schranke entstehen. Diese Flecken wären damit alleiniger Ausdruck solcher Gefäßregulationsstörungen ohne vitale Permeabilitätsstörung. Andererseits können diese Regulationsstörungen aber auch schon intra vitam zu Schrankenstörungen und damit zu einer Vitalfärbung i.e.S. geführt haben.

Unsere Experimente sagen über die zeitliche Reihenfolge von Schrankenstörung und Hirnvolumenvermehrung nichts Sicheres aus. Letztere stellten wir häufig am Druckkonus, bei starken Traumen auch an anderen Massenverschiebungen fest. Ebenso wie im Falle der Schrankenstörung bei anderen Formen der Hirnvolumenvermehrung, experimentellem Tumor, Entzündung usw. dürfte aber auch beim Hirntrauma keine einfache Kausalbeziehung in dem Sinne vorliegen, daß die Schrankenstörung die alleinige Ursache der Volumenvermehrung wäre.

Zur Stützung unserer a.a.O. näher begründeten Ansicht, daß für die Entstehung von Schrankenstörungen allgemein eine Verschiebung des Hirngewebs- $p_{\mathrm{H}}$ nach der sauren Seite verantwortlich zu machen ist, lassen sich im speziellen Falle des Hirntraumas einige, teilweise schon vor langer Zeit gemachte Feststellungen anführen: Bereits 1922 konnten Knauer und

6 H. B e cker, Dtsch. Z. Nervenheilkunde 164, 560 [1950].

7 A. Knauer u. E. Enderlein, J. Psychol. Neurol. 29, 1 [1922].
Enderlen ${ }^{7}$ diese Verschiebung nach experimenteller Commotio, relativ grob, mit Lackmuspapier nachweisen. Später zeigten Peters u. S elb a ch ${ }^{8}$, daß die Neutralisationsfähigkeit des Hirngewebes für saure Valenzen posttraumatisch abnimmt, schließlich fand V a ra-L o p e s ${ }^{9}$ beim Kaninchen bereits kurz nach Schädelhirntraumen im Liquor eine $p_{\mathrm{H}}$-Verschiebung zur sauren Seite. Als Ursache dieser Säuerung werden im allgemeinen die Gefäßregulationsstörungen und die in ihrem Gefolge auftretende Hypoxydose angesehen. Da diese in den Grenzzonen besonders stark sind, gewinnt von den beiden oben angeführten Deutungen der fleckigen Grenzzonenfärbung diejenige an Wahrscheinlichkeit, nach der es sich um eine intravitale Permeabilitätsstörung handelt. Auf den Mechanismus der Ausbreitung der Schrankenstörung und die Bedeutung der Beteiligung des Rückenmarkes sind wir in unserer oben genannten Arbeit näher eingegangen.

Abschließend möchten wir den auffallenden Befund einer erheblichen Störung der Bluthirnschranke beim Bagatelltrauma besonders betonen und auf die Möglichkeit seiner klinischen Bedeutung hinweisen. Es entsteht die Frage, ob und in welchem Umfange die klinischen Erscheinungen nach selbst „harmlosen“ Schädelhirntraumen auch beim Menschen mit Schrankenstörungen in Verbindung zu bringen sind. Wenn wir, wozu wir wohl berechtigt sind, annehmen, daß Störungen der Blut-Liquorschranke posttraumatisch in gleichem Umfange auftreten wie solche der Bluthirnschranke, so wäre es, gerade unter dem Aspekt der klinischen Bedeutung der Schrankenstörung, wünschenswert, die Prüfungsmethoden der Blutliquorschranke in der Klinik weiter auszubauen und in die Diagnostik der gedeckten traumatischen Hirnschädigungen einzubeziehen.

8 G. Peters u. H. Selb a ch, Arch. Psychiatrie 116, 531 [1943].

9 Vara-Lopez, Arch. klin. Chirurgie 150, 111 [1928]. 